\title{
XII. Ueber die Zwillingsbildung und den orientirten Schimmer am gediegen Eisen.
}

\author{
Von \\ G. Linck in Strassburg i/E. \\ (Mittheilungen aus dem mineral. Institut der Universität Strassburg, Nr. 17. \\ Fortsetzung von Bd. 19, S. 444.) \\ (Mit 3 Textfiguren.)
}

An dem Meteoreisen von Bra una u wurde im Jahre 1848 von Beinert*) wahrgenommen, dass geschliffene und mit Säure geätzte Platten in verschiedenen Richtungen von Linien oder Lamellensystemen durchzogen werden, welche er für Widmannstätten'sche Figuren hielt.

Ein Jahr später hat J. G. Neu mann **) dasselbe Eisen einer eingehenden Untersuchung unterworfen und dabei zweierlei nachgewiesen und in ausführlicher Weise begründet. Erstens nämlich: dass der ganze Meteorit ein einheitliches Krystallindividuum mit durchgehender hexaëdrischer Spaltbarkeit sei und ferner, dass jene von Beinert für Widmannstättensche Figuren erklärten Linien nicht einem lamellaren Aufbau nach dem Oktaëder entsprechen, sondern dass es Zwillingstracen seien, hervorgerufen durch den Zwillingsbau mehrerer nach Oktaëderflächen symmetrisch liegender und sich durchkreuzender Würfel. Neumann constatirte auf einer angeschliffenen und geätzten Wurfelfäche das Auftreten von sechs verschieden verlaufenden Liniensystemen und bestimmte deren gegenseitige Lage, sowie ihre Lage zu den Wurfelkanten. Er verfuhr dann ebenso auf anderen gegen den Würfel geneigten Schnittfächen und schloss aus seinen Untersuchungen, dass die Linien hauptsächlich den Tracen eines anderen zum Hauptwürfel in Zwillingsstellung stehenden Würfels entsprechen, dass

*) Be in ert, Meteorit von Braunau am 14. Juli 1847. Breslau 1848.

**) J. G. Neumann, Ueber die krystallinische Structur des Meteoreisens von Braunau. Nalurwiss. Abh. v. W. Haidinger 3, 2. Wien 1849.

Groth, Zeitschrift f. Kryatallogr. XX. 
aber untergeordnet auch noch Linien vorkommen, welche einem dritten, vierten und fünften nach anderen Oktaëderflächen symmetrisch gestellten Würfel angehören. Die Gesammtheit dieser Tracen entspricht auf einer Würfelfläche sechs Liniensystemen, von denen zwei den Diagonalen parallel gehen, die übrigen dagegen immer je eine Würfelecke mit den Mittelpunkten der gegenüberliegenden Kanten verbinden.

Neumann hat ferner an dem ungeätzten Spaltungsstuck ausser den Spaltungsfächen nach dem Hauptwürfel noch kleine glänzende Flächen beobachtet, von denen er vermuthet, dass sie Spaltungsflächen des in Zwillingsstellung befindlichen Hexaëders seien und gegenuber dem Hauptwüfel die Lage des „Trigonalikositetraëders $2 O$ « besitzen.

Diese Auffassung von Neumann ist späterhin allgemein angenommen worden und ist auch heute wohl noch uberall in Geltung, obgleich Durchkreuzungszwillinge mit derart regelmässigen Verwachsungsflächen, wie sie hier vorliegen muissten, bei den ubrigen Krystallen sich niemals wiederfinden.

Tscbermak*) hat später sowohl das Meteoreisen, als auch künstliches Eisen noch einmal eingehend untersucht und ist zu demselben Resultate gekommen wie Neumann. Auch er fand, dass die Flächen des zweiten, die Zwillingstracen bildenden Würfels die Lage des Triakisoktaëders $\{221\}$ gegentuber den Flächen des Hauptwürfels haben und begründet dies durch Messung der ebenen, von Zwillingstracen und Wurfelkanten eingeschlossenen Winkel auf der Würfelfläche. Die Ursache für diesen eigenthumlichen Bau glaubt er in dem molekularen durch Skelettbildung bedingten Aufbau gefunden zu haben und zieht zur Erklärung entsprechende Skelettbildungen des künstlichen Eisens heran.

Zweifel an der völligen Richtigkeit der Neumann'schen Auffassung sind aber wiederbolt aufgetaucht. So insbesondere bei G. Rose $e^{* *}$ ), wenn er schreibl: wandere balten diese Linien fur Anzeigen von versteckten Spaltungsflächen und allerdings würden die Durchschnitte des Hexaëders mit dem Ikositetraëder $\left(a: a: \frac{1}{2} a\right)$ oder dem Triakisoktaëder $\left(\frac{1}{2} a: \frac{1}{2} a: a\right)$ ganz dieselben Linien geben «. Er vergleicht weiterhin diese Linien, die er an einem Spaltungswürfel künstlichen Eisens in derselben Weise beobachtet hat, mit linienförmig an einander gereihten Aetzeindrücken. Die Linien sollten auch in ihrem Verlaufe auf an einander grenzenden Wurfelflächen meist dem Ikositetraëder 202 , seltener dem Triakisoktaëder $2 O$ entsprechen.

An dem küstlichen Eisen wurden die Linien schon im Jahre 1855 von

*) Tschermak, Das Krystallgefüge des Eisens, insbesondere des Meteoreisens. Sitzungsber. d. Wiener Akad. 70, I, 443 ff.

*) G. Rose, Beschreibung und Eintheilung der Meteoriten etc. Abhandl. d. kgl. Akad. d. Wiss. zu Berlin 1863. 
Prestel*) entdeckt und als Widmannstätten'sche Figuren gedeutet. Die Auffindung geschah an einem durch anhaltende Benutzung grobkrystallinisch gewordenen Roststabe $\left.{ }^{* *}\right)$.

S a d e b e ck ${ }^{* * * *}$ ) hat jenes oben erwähnte, als Geschenk von Mitscherlich an $\mathrm{R}$ os e gekommene, Eisenspaltungsstïck, welches die Linien schon mit blossem Auge recht deutlich erkennen liess, einer eingehenden Untersuchung unterworfen. Er fand durch goniometrische Messung den Winkel zwischen den glänzenden kleinen Flächen, deren Tracen auf der Würfelfläche diagonal verlaufen, und der Würfelfläche, auf welcher sie auftreten, zu 144.10. Da er weiter mit Neumann und Tschermak annimmt, dass diese kleinen Flächen Spaltungsflächen der Zwillingslamellen nach dem Wtirfel darstellen, so kommt Sa debeck zu dem Resultate, dass die beiden Würfel nicht symmetrisch nach $O$ liegen können, weil dann der gemessene Winkel $109^{\circ} 28^{\prime}$ betragen müsste, entsprechend der Lage der Flächen von $2 O$ am Würfel. Er nimmt deshalb diejenige Fläche als Zwillingsebene an, welche in der Zone der Diagonale des Würfels liegt und den Winkel von $144 \frac{1}{4}^{0}$ halbirt, d. i. die Fläche $\left(a: \frac{9}{20} a: \frac{9}{20} a\right)$ und sagt: dem Würfel sind Zwillingslamellen nach dem Gesetze: Zwillingsebene eine Fläche von $\frac{20}{9} O$, eingeschaltet.

Das hiesige mineralogische Institut hatte vor einigen Jahren Gelegenheit, durch Tausch in den Besitz eines kleinen Spaltungsstückes von künstlichem Eisen zu gelangen. Es ist ein Theil eines unregelmässigen rundlichen Stiuckes, welches aus einer Eisenschlacke stammt, und ist ausser der unregelmässigen Oberfläche begrenzt von drei aneinander stossenden Würfelflächen von 10,7 und $6 \mathrm{~mm}$ Kantenlänge. Es umschliesst einige runde Tropfen von grünlichbrauner Schlacke, welche stark zersprungen ist.

Die Spaltungsflächen sind glänzend und lassen sehr deutlich schon mit blossem Auge obne Aetzung die bekannten Neumann'schen Linien erkennen; diese treten in manchen Richtungen in solcher Menge auf, dass man in eben diesen Richtungen nach beiden Seiten einen sehr deutlichen orientirten Schimmer wahrnimmt.

Auf jeder Würfelfläche lassen sich sechs Systeme von Linien wahrnehmen, die den beiden Diagonalen der Würfelfläche und den Verbindungsecken mit den Mittelpunkten der gegenüberliegenden Kanten parallel gehen. Nicht alle Systeme sind gleich stark geschaart, sondern bald ist die eine, bald die andere Richtung besonders deutlich ausgeprägt, bald ist auch ein

*) Haidinger, Bemerkungen über die zuweilen im geschmeidigen Eisen entstandene krystallinische Structur etc. Sitzungsber. d. Wiener Ak. 1855, 15, $354 \mathrm{ff}$.

**) Eine solche nachträgliche Umlagerung wird bekanntlich neuerdings von den Technikern für unmöglich gehalten.

***) S a debeck, Ein neues Zwillingsgesetz im regulären Systeme, beobachtet am gediegen Eisen. Poggendorff's Annalen 156, 554. 
System nur auf einem eng begrenzten Raume stark entwickelt. Am meisten springen stets die diagonal verlaufenden Systeme in die Augen.

Die Breite dieser Linien ist ausserordentlich gering, selbst mit der Lupe sieht man nur einen dünnen Strich, der jedenfalls nicht uber $\frac{1}{10} \mathrm{~mm}$ breit ist. An vielen Stellen sieht man statt der Linie eine papierdünne Lamelle, welche aus dem Eisen hervorragt. Sie steht dann ubrigens nie mehr als um Bruchtheile eines Millimeters uber die sonst einheitliche Spaltfläche des Würfels vor. Bricht man das Blättchen ab, so verbleibt auf der Spaltfläche eine zarte dunkele Linie.

Bei näherer Untersuchung stellt sich eine Spaltungsfläche des Wúrfels so dar, dass sie allüberall senkrecht auf die Richtungen der Liniensysteme treppenförmig absetzt. Zwischen je zwei in verschiedenem Niveau liegenden Theilen der Wurfelfläche leuchtet im reflectirten Lichte eine kleine, glänzende Fläche auf, welche gleichzeitig einspiegelt mit den demselben Liniensysteme angehörigen hervorstehenden Lamellen und unter grösserem oder geringerem Winkel bald nach der einen, bald nach der anderen Seite geneigt ist, derart, dass jedem Systeme zwei entgegengesetzt, aber unter

Fig. 1 .

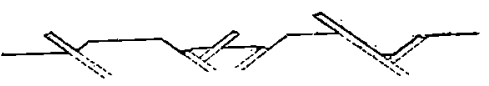
gleichem Winkel geneigte Flächen angehören. Die beistehende Fig. 1 giebt ein schematisches, vergrössertes Bild einer Würfelfläche in Projection auf eine zu ihr senkrecht stehende Fläche von

$\{110\}$. Dieselbe Erscheinung beobachtet man mit Bezug auf alle Liniensysteme.

Die kleinen Flächen sind so stark glänzend, dass sie auf dem Reflexionsgoniometer bei Anwendung des Websky'schen Spaltes als Signal wenn auch lichtschwache, so doch gute Reflexe geben, während die Würfelfläche einen breiteren hellen Fleck, meist aber mit deutlichem Culminationspunkte liefert. Es war deshalb möglich, die zwischen den drei Wurfelflächen und den jeweils auf ihnen auftretenden kleinen Flächen, welche in der Zone der Diagonalen liegen, zu messen. Dasselbe gelang auch mit Bezug auf je eine Würfelfläche und je zwei mit ihr in einer Zone liegenden kleinen Flächen, welche der Zone der Diagonalen der benachbarten Spaltungsflächen angehören. Die Einstellung der letzteren Zonen wurde stets so am schnellsten bewirkt, dass die betreffende Würfelfläche und eine auf ihr auftretende, von Wurfelecke zum Mittelpunkte einer gegenüberliegenden Kante verlaufende Linie justirt wurden. Es waren dann stets die kleinen Flächen der Diagonalzone der anliegenden Würfelfläche fast genau justirt. Somit konnten alle zwölf Flächen, welche den auf den Wurfelflächen beobachteten sechs Tracen entsprechen, in die Messung einbezogen werden.

Die in der Zone der Diagonale einer Wurfelfläche liegenden kleinen Flächen bilden mit jener Normalen-Winkel, welche bei zwölf verschieden 
liegenden kleinen Flächen elf Mal Werthe lieferten, welche zwischen $35^{0} 54^{\prime}$ und $35010^{\prime}$ schwankten. Ein einziges Mal wurde, wohl in Folge schlechter Lichtbilder, der Werth $34^{0} 36^{\prime}$ gefunden. Aus jenen elf Messungen folgt als Mittel $35^{0} 26^{\prime}$.

Fưr die anderen Zonen ergeben sich je zwei Winkel, nämlich für den Winkel zwischen zwei benachbarten kleinen Flächen sechs Werthe, welche von $48^{\circ} 1^{\prime}$ bis $48^{\circ} 57^{\prime}$ schwanken und als Mittel $48^{\circ} 33^{\prime}$ liefern, ferner bei schlechteren Reflexen die Winkel $47^{\prime} 4^{\prime}, 47^{\circ} 45^{\prime}, 49^{\circ} 32^{\prime}, 49^{\circ} 13^{\prime}$ und $49^{\circ} 12^{\prime}$ - für den Winkel zwischen einer Wurfelfläche und der benachbarten kleinen Fläche waren in Folge des breiten Lichtbildes der Wurfelfläche genaue Einstellungen nicht möglich. Die vier besten Werthe schwanken zwischen $65^{\circ} 12^{\prime}$ und $66^{\circ} 39^{\prime}$ und liefern als Mittel $65^{\circ} 46^{\prime}$. Die übrigen Werthe gehen herab bis zu $64^{\circ} 36^{\prime}$ und hinauf bis zu $67^{\circ} 46^{\prime}$.

Aus den Messungen ergiebt sich, dass die kleinen Flächen in ihrer Lage sämmtlichen Flächen des Ikositetraëders $\{112\} 202$ entsprechen. Die zuerst gemessenen Zonen werden - wenn wir das Spaltungsstuck so aufstellen, dass die drei Spallungflächen die Symbole (001), (100) und $(0 \bar{T} 0)$ erhalten - gebildet von den Flächen (1T2), (001), (T12) resp. (112), (001), (TT2) resp. (211), (100), (2TT) resp. (2TT), (100), (21T) resp. (1 $\overline{2} 1),(0 \bar{T} 0)$,

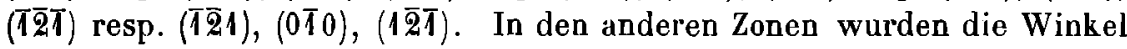
zwischen folgenden Flächen gemessen: $(001),(211),(21 T)-(2 T 1),(2 T T)$,

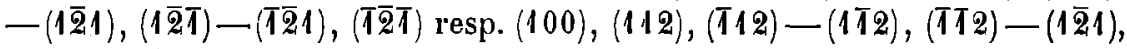
$(\bar{T} \overline{2} 1)-(1 \overline{2} T),\left(\bar{T}_{\overline{2}} \mathrm{~T}\right)$ resp. $(0 \bar{T} 0),(1 \bar{T} 2),(112)-(\bar{T} \mathrm{~T} 2),(\bar{T} 12)-(2 \mathrm{~T} 1),(211)$ $-(2 T \bar{T}),(21 T)$.

Die hieraus berechneten Winkel mögen hier neben den gefundenen Platz finden.

\begin{tabular}{|c|c|}
\hline Berechnet: & Gefunden : \\
\hline$(001):(112)=35^{0} 16^{\prime}$ & $35^{0} 26^{\prime}$ \\
\hline$(112):(T 12)=48 \quad 11$ & 4833 \\
\hline$(001):(211)=6554$ & 6546 \\
\hline
\end{tabular}

Aus dem Vorhergehenden folgt nun zunächst, dass bei der Voraussetzung, die kleinen Flächen seien Spaltungsflächen des in Zwillingsstellung befindlichen Würfels, die beiden Wurfel sich nicht, wie Neumann und Tschermak annehmen, in Zwillingsstellung nach einer Oktaederfläche befinden können, sondern sie müssen, wie $\mathrm{S}$ ad ebeck angiebt, symmetrisch liegen mit Bezug auf die Fläche eines Triakisoktëders.

Aber auch Sadebeck hat nicht das Richtige getroffen, weil die Voraussetzung, die fraglichen kleinen Flächen seien Spaltungsflächen des in Zwillingsstellung befindlichen Würfels, nicht zutrifft. Aus meinen Ausfuhrungen auf voriger Seite ergiebt sich, dass längs dieser Flächen die Zwillingslamellen im Eisen stecken und jene somit Absonderungsflächen sind. 
Wären sie Würfelflächen, so müssten sie beiderseitig von Zwillingstracen begrenzt sein, was nie zu beobachten war.

Die Zwillingslamellen sind dem Eisen also eingelagert nach sämmtlichen zwölf Flächenpaaren von \{112\} 202. - Auf nebenstehender Fig. 2

Fig. 2.

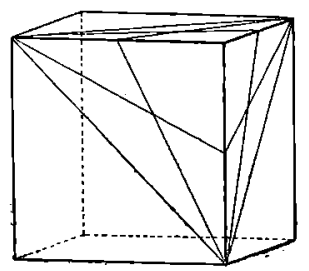
ist die Lage dieser Ebenen mit Bezug auf eine Würfelecke dargestellt. - Ueber die krystallographische Orientirung jener Lamellen haben wir jedoch keinen directen Anhaltspunkt; wir werden aber nicht fehl gehen, wenn wir annehmen, sie seien symmetrisch nach $\{112\}$ eingelagert, weil dies der einfachste und bei anderen Mineralien öfters beobachtete Fall ist. Es ergiebt sich daraus für das Eisen das Zwillingsgesetz :

"Zwillingsebenen die Flächen von $\{112\} 202 . "$

Man könnte dieses „Gesetz allerdings auch ausdrücken: "Zwillingsebenen die Flächen des Oktađders", weil jede Fläche des Ikositetraëders \{112\} 202 auf einer Oktaëderfläche und mit ihr auf der Symmetrieebene $\infty 0$ senkrecht steht. Von Durchkreuzungszwillingen kann aber keinenfalls die Rede sein; man müsste dann vielmehr hinzuselzen: "Verwachsungsflächen die Flächen von $\{112\} 202 . "$ "

Die kleinen glänzenden Flächen sind Absonderungsflächen in Folge der Zwillingsbildung.

Was die Entstehung dieser Zwillingslamellen betrifft, so haben wir bis jetzt żwei Hypothesen: die eine von Tschermak nimmt eine Entstehung während der Krystallisation des Eisens an; die zweite von Sadebeck führt die Entslehung auf die Erschutlerung des Eisens beim Bruche zurúck, derart, dass $\{112\}$ Gleitlächencharakter hätte.

Wir wissen nun, dass das Meteoreisen von Braunau in grosser Menge Rhabditnadeln parallel den Würfelflächen eingelagert enthält. Sind die Zwillingslamellen primär, so müssen diese Krystallnadeln entweder an den Lamellen aufhören oder sie müssen ununterbrochen durch dieselben hindurchgehen. An einer geätzten Würfelfäche des genannten Eisens habe ich die Beobachtung gemacht, dass man etwa bei der Hälfte der Nadeln, insbesondere bei den dünnsten derselben, uberall die Brüche beobachten kann, wo sie eine Zwillingstrace uberschreiten, während die andere Hälfte, und unter diesen besonders die dickeren Nadeln, solche Brtuche nicht erkennen lässt. Daraus scheint mir zu folgen, dass wir es thatsächlich mit Zwillingen in Folge Gleitung zu thun haben, dass also die Zwillingsbildung hervorgerufen ist durch secundäre Vorgänge (Erschütterung, Spaltung) oder bei der Erkaltung. Bei den dickeren Rhabditnadeln mag das Eisen der Nadel ausgewichen sein, weil die Kraft nicht genugte, um die Nadeln zu zerbrechen. 
Das eben erwähnte Verhalten der Rhabditnadeln beobachtet man nur deutlich auf geätzten Flächen. Diese zeigen aber dann noch andere Erscheinungen in deutlicher Weise. So sieht man einerseits die von Tschermak erwähnten Aetzgrubchen in Form von vierseitigen, den Würfelkanten parallelen Vertiefungen mit sebr steilen Seitenflächen. Andererseits beobachtet man, dass die Zwillingslamellen viel schneller gelöst werden, so dass sie sich in kurzer Zeit als vertiefte Furchen darstellen, die aber alle eine ganz eigenthumliche Gestaltung zeigen. Es bildet sich nämlich bei jeder Lamelle eine schief liegende, unebene Fläche, welche möglicher Weise gegen die Hauptspaltungsfläche nach der die Trace bildenden Fläche von \{112\} symmetrisch gestellte Würfelfläche des Zwillings sein könnte, und dadurch wird die lebhaft schimmernde Zwillingsfläche frei gelegt, wie es beistehende Fig. 3 schematisch zeigt. Durch diese so freigelegten Zwillingsflächen wird der orientirte Schimmer der hexaëdrischen Meteoreisen hervorgerufen und nicht durch die Aetzfiguren, wie Ts chermak*) annimmt. Die Stärke des Schimmers ist lediglich bedingt durch die Breite und die Häufigkeit der Zwillingslamellen. Bei dem mir vorliegenden kunstlichen Eisen kann der Schimmer schon sehr deutlich ohne Aetzung wahrgenommen werden und zwar in den Richtungen aller zwölf Ikositetraëderflächen. Am deutlichsten wird man ihn am Würfelspaltungsstücke stets in den Zonen der Diagonalen wahrnehmen, weil die zugehörigen Ikositetraëderflächen am flachsten gegen die Wüfelfläche geneigt sind.

So zeigte z. B, ein in der hiesigen Sammlung befindliches Stuck Meteoreisen von Fort Duncan in Texas orientirten Schimmer nach mindestens fünf verschieden liegenden Flächen. Der Winkel zwischen zwei solchen Flächen, die derselben Zwillingstrace angebören, wurde durch Schimmermessung goniometrisch bestimut und zu annähernd $72^{\circ}$ gefunden, also nahe ubereinstimmend mit dem Winkel $(112):(\bar{\top} \bar{T} 2)=70^{0} 32^{\prime}$. Ausserdem war auch directe Orientirung des Schimmers gegen die, an dem Eisen hergestellten, Spaltungsflächen möglich, so dass ein Zweifel über die Ursache des Schimmers nicht mehr bestehen kann.

Mineralogisches und petrographisches Institut der Universität Strassburg, im Januar 1892.

*) I. c. Vergl. auch T schermak, Lehrbuch der Mineralogie. 3. Aufl., 1888. 